\title{
Max-Min Fair Link Quality in WSN Based on SINR
}

\author{
Ada Gogu, ${ }^{1}$ Dritan Nace, ${ }^{2}$ Supriyo Chatterjea, ${ }^{3}$ and Arta Dilo ${ }^{3}$ \\ ${ }^{1}$ Polytechnic University of Tirana, 1010 Tirana, Albania \\ ${ }^{2}$ Université de Technologie de Compiègne, 60205 Compiègne Cedex, France \\ ${ }^{3}$ University of Twente, 7500 AE Enschede, The Netherlands \\ Correspondence should be addressed to Ada Gogu; agogu@fti.edu.al
}

Received 5 March 2014; Revised 21 May 2014; Accepted 23 May 2014; Published 23 June 2014

Academic Editor: Michał Pióro

Copyright (C) 2014 Ada Gogu et al. This is an open access article distributed under the Creative Commons Attribution License, which permits unrestricted use, distribution, and reproduction in any medium, provided the original work is properly cited.

\begin{abstract}
This paper addresses first the problem of max-min fair (MMF) link transmissions in wireless sensor networks (WSNs) and in a second stage studies the joint link scheduling and transmission power assignment problem. Given a set of concurrently transmitting links, the MMF link transmission problem looks for transmission powers of nodes such that the signal-to-interference and noise ratio (SINR) values of active links satisfy max-min fairness property. By guaranteeing a "fair" transmission medium (in terms of SINR), other network requirements may be directly affected, such as the schedule length, the throughput (number of concurrent links in a time slot), and energy savings. Hence, the whole problem seeks to find a feasible schedule and a power assignment scheme such that the schedule length is minimized and the concurrent transmissions have a fair quality in terms of SINR. The focus of this study falls on the transmission power control strategy, which ensures that every node that is transmitting in the network chooses a transmission power that will minimally affect the other concurrent transmissions and, even more, achieves MMF SINR values of concurrent link transmissions. We show that this strategy may have an impact on reducing the network time schedule.
\end{abstract}

\section{Introduction}

Wireless sensor networks (WSNs) are presently used in a wide range of applications. They are usually deployed as a standalone system or as part of a larger, more sophisticated system, such as the Internet-of-Things. However, this technology has stringent requirements mainly related to energy and wireless transmission medium. The power allocated to sensor nodes in a network is fundamentally constrained; nonetheless they have to transmit their data which costs sufficient energy. Because of interference, the concurrent wireless transmissions may be easily corrupted, which increases the number of packet retransmissions. This may cause energy depletion and delays in the network. Existing solutions such as transmission power control and blacklisting PCBL [1], adaptive transmission power control ATPC [2], and adaptive and robust topology control ART [3] use parameters like received signal strength indicator (RSSI) or packet receive rate (PRR) to evaluate the quality of a link. However, RSSI or PRR may not capture the effect of interference in particular scenarios as will be further detailed.
In addition, we consider the SINR parameter and solve the problem of fair SINR link transmission. Hence, for a set of activated links, the problem seeks to find the power of the transmitting nodes such that the SINR value is fair at each receiver. Fairness is a key consideration in WSN scenarios in order to maintain a balanced view of the network and, in this case, to give the same priority to each of the concurrent transmissions. This increases the number of potential concurrent links scheduled in the same time slot and therefore reduces the schedule length. However, the feasibility of this problem is tightly coupled with the given set of activated links and therefore the scheduling. This is a typical example that shows how the optimization problems in WSN may often lead to cross-layer ones. The cross-layer optimization problem comes to be the joint link scheduling and power assignment (JLSPA) that seeks to find an efficient link scheduling scheme, in which the power of sender nodes is set variable, such that certain requirements are satisfied.

In a time-driven WSN, sensor nodes need to send their data periodically according to a regular traffic pattern. This period is usually known as a round of data gathering. 
The time needed to perform a round will be determined by the schedule length which, on the other hand, is constrained by the interference effect. The relation between these two parameters is further detailed in Sections 2 and 3.1. In this work, interference is taken into account by considering the SINR parameter. Hence the subject of our research is (i) to find a power allocation scheme which guarantees a max-min fair SINR and (ii) to solve the JLSPA problem. We design a solution for two scenarios that are slightly different: the transmission power of a node changes per slot within a frame; the power is fixed (the same) in a frame level.

The paper is organized as follows. In Section 2 we recall first the interference definition and the related SINR constraint, and we present a review of related works regarding the JLSPA problem and the power assignment schemes. The fair SINR link transmission problem is introduced in Section 3. Here we state in more detail the research motivation, the problem definition, and the mathematical model. In Section 4 we present an (centralized) approach for solving the MMF SINR link transmission problem. Next, we discuss a variant of this problem with constant powers in Section 5. In Section 6 the JLSPA problem is considered and numerical results are presented to show the performance of the method discussed in the previous section. Finally, we conclude this work in Section 7.

\section{Related Work}

Application of optimization theory to the design of WSN algorithms is addressed in different works and for a summary we refer the reader to [4]. Regarding MMF formulation of the problems, they are generally employed to ensure fairness related to link rates $[5,6]$ or end-to-end flows $[7,8]$. MMF is applicable in numerous areas where it is desirable to achieve an equitable distribution of certain resources shared by competing demands and is therefore closely related to max-min or min-max optimization problems [9]. In this paper, our focus is on the SINR max-min fairness. Generally speaking, a vector of transmission links is said to be MMF with respect to SINR if the corresponding vector of SINR values is MMF; that is, one cannot increase the SINR value of some transmission link without decreasing the SINR value of some other links with lower SINR.

As far as the scheduling problem apart is strongly concerned with interference avoidance for achieving successful multiple concurrent transmissions, the key point is the interference definition. Two basic definitions can be distinguished: the protocol and the physical one. The protocol definition of interference assumes that two links, which are less than $k$ hops $(k \geq 1)$ away from each other, interfere potentially and cannot be scheduled in the same time slot. The indicated number of hops refers to the number of hops between the sender nodes of these links. On the other hand, the physical definition is based on the SINR constraint where the transmission links that do not satisfy the SINR constraint cannot be scheduled simultaneously (in the following we will use interchangeably the terms transmission links and links).
However, as we will later see, the problem remains $\mathscr{N} \mathscr{P}$ hard regardless of the interference definition. Following the protocol definition of the SINR, the JLSPA seeks to find a minimum-length schedule for all nodes in the network such that they do not interfere with each other. In the simple case $(k=1)$, the constraint requires that two edges in the same time slot do not share a node. The scheduling problem is widely modeled as the well-known optimization problem of graph coloring in which one seeks to find the minimal number of colors (chromatic number) necessary to color a graph such that no two adjacent nodes (two nodes are considered adjacent if they are " $k$ " hops away from each other) have the same color. Finding the chromatic number in a graph is $\mathscr{N} \mathscr{P}$-hard; therefore different methods have been proposed for this problem by the Operation Research Community. These methods have been adapted and implemented for WSN; see, for instance, [10-13].

As the protocol model underestimates the number of successful multiple concurrent transmissions, different works $[1,14,15]$ consider the cumulative interference proposed by the SINR model. For solving the link scheduling problem under physical interference, one approach seeks to classify the links according to their length (or distance). Hence, in [15-17] each link belongs to a class $C_{k}$ if its length $l_{i}$ is $2^{k} \leq\left|l_{i}\right|<2^{k+1}$. The idea behind the partition of links in classes is to schedule at the same time the links with the same length or very different one. The complexity of the proposed algorithm is $O\left(\log ^{4} n\right)$ where $n$ represents the number of nodes in the network. Kesselheim [18] identifies another condition that the set of links scheduled in the same time slot should satisfy. Given two links, this condition is related to the ratio between the distance of one link and the respective distance between the transmitter of this link and the receiver of the other one. The scheduling algorithm is a greedy one, which ensures that the condition is satisfied if another link is added to the set of links scheduled in a given time slot. Goussevskaia et al. [16] design greedy heuristic scheduling. For each class $C_{k}$, they partition the network area into squares of side length $a \cdot 2^{k}$, where $a$ is a constant, and color the squares using 4 colors. Next, for each color they pick up the links having their receiver in different squares and assign them to a time slot. The process is repeated till all the links are scheduled. The same idea is revisited in [17]. In addition, they consider the case when the links have different demands to satisfy. Moreover, the length of the time slots is not fixed and the links may be scheduled more than once in a frame. In order to identify the links that can be scheduled simultaneously, [17] proposed a link classification based on the signal to noise ratio (SNR) value. Instead of using the SINR threshold, Santi et al. [17] consider a graded SINR model which relates the PRR with SINR values as in Figure 1. Their algorithm computes a schedule length of $O(r \cdot t)$, where $r$ is the maximal number of receivers in a cell and $t$ is the time needed to transmit with the minimal SINR estimated in the frame (the time for transmitting a data unit is $t=1 / f$ where $f$ is the data rate computed according to Shanon's channel capacity formula $f=B \cdot \log _{2}(1+$ SINR), where $B$ is the channel bandwidth). To identify the set of concurrent 


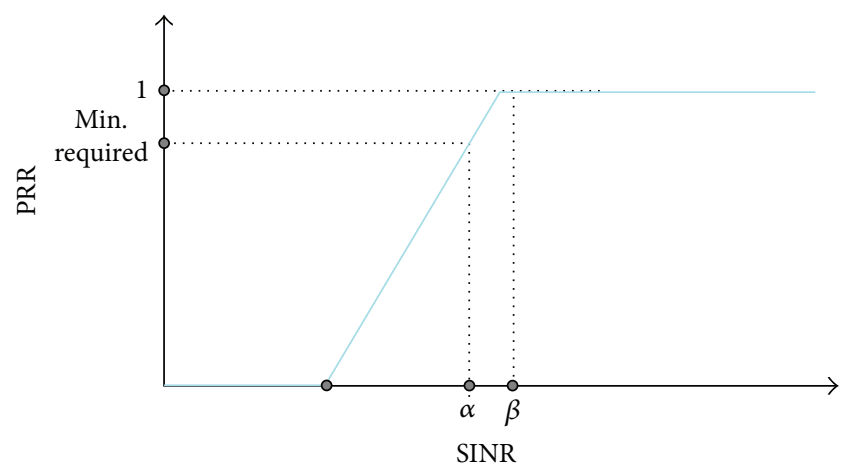

FIGURE 1: SINR graded model. $\alpha$ and $\beta$ are the lower and upper bounds of the desired SINR value, respectively, as described in (2) and (4).

links, another class of algorithms solves the maximum link matching problem which seeks to find the maximum number of links in a given graph that do not have a node in common [19].

Despite the existence of different approaches for solving the scheduling problem under SINR constraint, designing a network protocol that takes this constraint into consideration is not trivial.

Till now, we have discussed the scheduling problem without emphasizing the power assignment strategy, which is of paramount importance. Based on the above discussed problems we classify these strategies into three main groups.

Uniform Power Assignment. This is the simplest and the most intuitive case where it is assumed that all the nodes use the same power to transmit their data. Hence the question is to find the optimal transmission range that maximizes the number of multiple successful concurrent transmissions [20].

Linear Power Assignment. This scheme uses the rule of assigning the power proportionally [17] to the signal attenuation (the simplest model of signal attenuation is given by $d^{\gamma}$ where $d$ is the length of the link and $\gamma$ the path loss exponent) which corresponds to the minimum power of transmission that guarantees a successful packet decoding from the receiver part. Similarly, the square-root power assignments, proposed by [21], assign the power proportionally to $\sqrt{d^{\gamma}}$. The linear and the uniform strategies are frequently used in MAC layer protocols.

Nonlinear Power Assignment. According to this strategy the power is disproportional to the link distance. The study of Moscibroda and Wattenhofer [15] shows that the uniform and linear power assignment may lead to inefficient scheduling as the shortest links may "suffer" due to the high power signals emitted by the sources of the longest ones. Hence, in [15] a nonlinear power assignment strategy which gives priority to the short links is proposed. It assigns a minimal power to the longest links such that the communication is feasible and next it increases with a scaling factor the power assigned to the shorter links. Using a different scaling factor, the same scheme is applied also in [18].

This work follows on from some recent work on power assignment [22]. In this study we go further and investigate max-min fair link quality among the active transmission links.

Related to the complexity of the problem of joint scheduling and power assignments, different variants of the problem are considered. Let us first refer to the link assignment problem. This problem needs to assign the links to different time slots such that two adjacent links will not be in the same time slot and the SINR constraints will be met for each of them. It is shown in [23] that this problem is at least as hard as the edge coloring problem and is thus $\mathcal{N} \mathscr{P}$-hard. Further, the problem of determining a minimum-length schedule that satisfies the SINR constraints is studied in [16]. By constructing a geometric instance of the scheduling problem, Goussevskaia et al. [16] show that the problem is reducible to the partition problem (given a set of integers, the partition problem seeks to decide whether it is possible to divide this set into two subsets, such that the sums of the numbers in each subset are equal). The case when the schedule has to satisfy the links demands (or flow rates) is shown to be $\mathscr{N} \mathscr{P}$-hard by reducing it to the matching problem [24]. Hence, different variants of this problem and their respective complexities are discussed in the literature. The proof of the complexity of our JLSPA problem is presented in the work of Katz et al. [25]. The JLSPA problem was shown to be $\mathcal{N} \mathscr{P}$-hard by [25]; when the network is embedded in the Euclidean plane, the power is variable and there are known upper and lower bounds on the power levels that can be used. Moreover, the proof remains true even for the case in which the sender node may choose its transmission power from an available set of discrete values.

\section{Problem Definition}

3.1. Research Motivation. For getting insights into modeling the power assignment problem under SINR constraints, we refer to some experimental test provided in [1-3]. Considering only one transmitter node and one receiver node and factoring out the issue of interference, it can be stated that the link quality between the transmitter and receiver improves as the transmitter increases its transmission power. This reasoning also holds for very sparse network where the transmitter is only within the range of the intended recipient but not in the range of any other nodes that may be simultaneously receiving data from other transmitting nodes. Under these circumstances, existing transmission power control protocols, such as those found in PCBL [1], ATPC [2], and ART [3], could help to ensure that an appropriate transmission power is used to achieve reliable link quality using minimum energy. These schemes generally use parameters such as the received signal strength indicator (RSSI) or packet receive rate (PRR) to evaluate the quality of a link. This information is then used to take decisions about the transmission power that should be set to maximize link quality using the least amount of energy. However, as the network density increases and every transmitting node is potentially within range of multiple 
receivers, interference plays a much larger role. Under such circumstances parameters such as RSSI or PRR do not give a good indication of whether link quality is poor or more importantly why it is poor (both power transmission and interference can be the cause). For example, if we assume that interference does not exist, higher RSSI reading generally translates into a higher PRR [1]. However, as interference increases, a higher RSSI may not result in a higher PRR, as the increased RSSI may be due to other nodes that are transmitting simultaneously and are within the range of the receiver. In addition, techniques like PCBL, ATPC, and ART only depend on information available locally at a node to make deductions about the quality of a link. As it can be seen from the performance of ART, a node may not always be able to accurately differentiate between packet loss due to a weak signal and that due to interference by using a localized approach. But as all nodes act independently of each other, one of drawbacks of such schemes is that nodes try to outdo each other. This results in higher power consumption and also has a detrimental effect on link quality. Due to these reasons, in this study we aim to find an optimal solution for transmission power assignment in a fair manner, using a centralized approach. Each node that is actively transmitting in the network chooses a transmission power that minimizes the interference effects on all the nondestination receivers. Our scheme aims to optimize the SINR parameter instead of only addressing RSSI or PRR as it is able to capture information about both the signal strength and interference more accurately.

3.2. Notation and Problem Definition. We model the wireless sensor network through a directed graph $G(V, E)$ where $V$ is the set of nodes representing the sensors and $E$ is the set of links representing the wireless channel communication between the sensors. For each link $(i, j) \in E, i$ indicates the transmitter node and $j$ the receiver one. The weight of link $(i, j)$ is denoted by $\omega_{i j}$ and represents the attenuation of the signal. In some other context, $\omega_{i j}$ may be referred to as gain if it would present the signal amplification to reach the receiver. We now assume that, in a given time, only a subset of links $M$ $(M \subset E)$ is activated. Let us denote by $\mathrm{TX}_{M}$ the subset of $V$ containing the heads (transmitting nodes) of the directed links $(i, j) \in M$ and by $\mathrm{RX}_{M}$ the tails (receivers nodes) of links in $M$.

Two properties can be noticed for $\mathrm{RX}_{M} / \mathrm{TX}_{M}$ subsets:

(1) $\mathrm{RX}_{M} \cap \mathrm{TX}_{M}=\varnothing$,

(2) $\mathrm{RX}_{M} \cup \mathrm{TX}_{M} \subseteq V$.

The $\operatorname{SINR}_{j}$ value estimated in the receiver $j$ according to [26], where $(i, j) \in M$, is given by

$$
\operatorname{SINR}_{j}=\frac{P_{i} / \omega_{i j}}{\sum_{k \in \mathrm{TX} \backslash\{i\}}\left(P_{k} / \omega_{k j}\right)+N_{a}},
$$

where $j \in \mathrm{RX}, P_{i}$ and $P_{k}$ are the power assigned to the sender nodes, $\omega_{i j}$ denotes the weight of the transmission link $(i, j), P_{k} / \omega_{k j}$ measures the interference of the other links over the receiver node $j$ of the link $(i, j)$, where $(i, j) \in M$, and $N_{a}$ is the floor noise which is considered as constant. Next, we define explicitly the parameters of a successful transmission. Clearly, a crucial parameter for estimating the link quality is PRR. This parameter is strongly related to SINR $[17,27]$. According to these works, the packets are successfully received only when SINR exceeds a given threshold. The graded SINR model graphically presented in Figure 1 shows the relation between PRR and SINR which is used in this work.

3.3. Mathematical Modeling. Given a set of concurrently transmitting links, the max-min fair link transmissions problem determines the transmission power allocated to nodes such that the SINR values of active links are MMF. For solving this problem, we will refer to a subproblem which is modeled as max-min linear programming. Let us present in detail the constraints and the objective function for this subproblem. First, in order to have fair link transmissions, we aim to maximize the minimum SINR value associated with receiver nodes. Moreover, this objective permits improving the quality of the worst link which usually comes out to be a key point for measuring the network performance. Let us have a look at the constraints.

(1) To have a successful transmission we require the SINR value at the receiver to be bigger than a threshold. We denote by $\alpha$ this lower bound as given in

$$
\operatorname{SINR}_{j}=\frac{P_{i} / \omega_{i j}}{\sum_{k \in \operatorname{TX} \backslash\{i\}}\left(P_{k} / \omega_{k j}\right)+N_{a}} \geq \alpha, \quad j \in \mathrm{RX}_{M} .
$$

(2) The intended signal strength measured by received signal strength indicator (RSSI) has to be bigger than a threshold RSSI ${ }_{0}$. This threshold represents the lowest power level of the signal which permits a receiver to detect and decode the information of the signal. It is also known as the receiver sensitivity and can be easily found in the data sheet of the radio transceiver:

$$
\operatorname{RSSI}_{i j}=\frac{P_{i}}{\omega_{i j}} \geq \operatorname{RSSI}_{0}, \quad(i, j) \in M,
$$

where $\operatorname{RSSI}_{i j}$ is the received strength indicator at the node $j$ when the link $(i, j) \in M$ is activated.

(3) Considering the graded SINR model in Figure 1, we can observe that beyond a given threshold $\beta$ of SINR, the PRR does not change. It is reasonable to keep the SINR values as close as possible to the $\beta$ value. Imposing $\beta$ as an upper bound for the SINR of all receivers has the high risk of infeasible solutions. Instead, we add the constraint for the lowest SINR as follows:

$$
\min _{j \in \mathrm{RX}} \operatorname{SINR}_{j} \leq \beta .
$$

\section{Max-Min Fair SINR Link Transmission}

We investigate in this section the problem of max-min fair SINR link transmission (MMFSLT). With respect to 


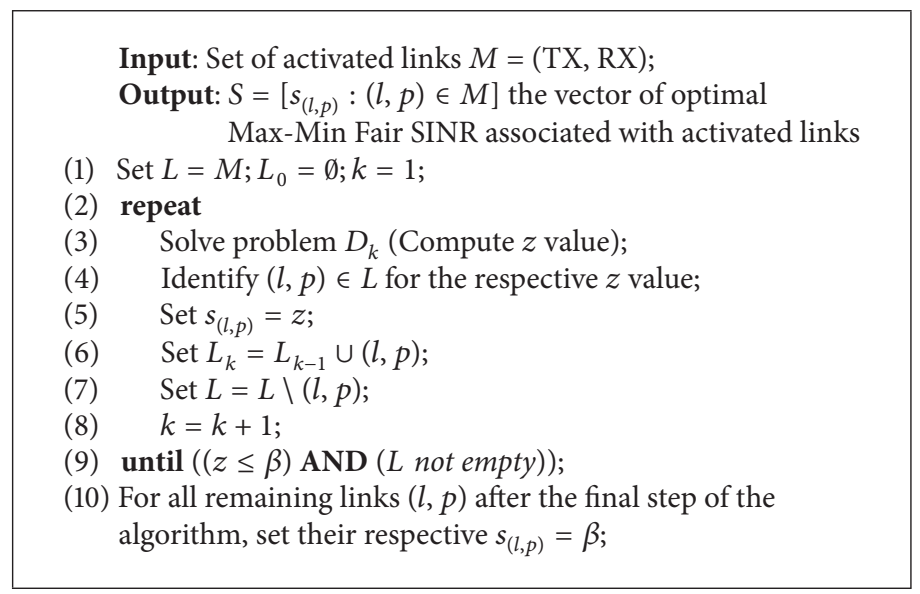

Algorithm 1: MMF algorithm (optimal max-min fair SINR link transmission).

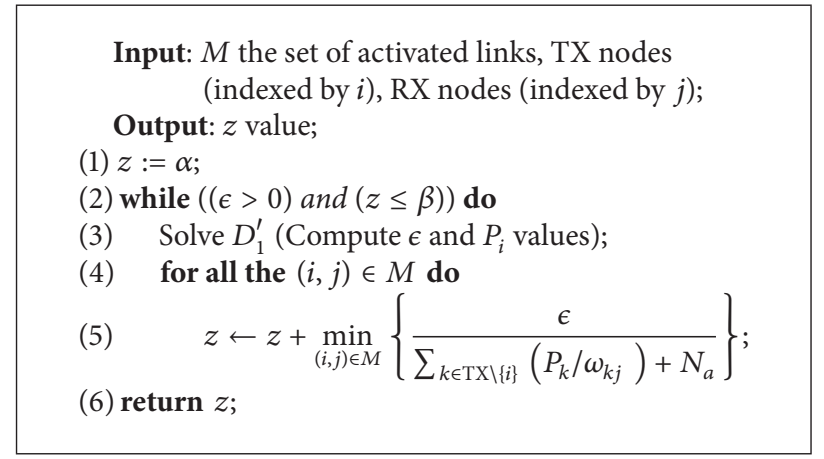

Algorithm 2: Max-min SINR.

the JLSPA problem, by looking for optimal and fair link transmissions strategies under SINR constraints, we aim to guarantee successful transmissions and incidentally reduce the schedule length. In fact, by guaranteeing MMF SINR, the number of potential concurrent links increases, which in turn implies shorter schedule length (see also Section 3). Hence, we present Algorithm 1 which solves the MMFSLT problem in Section 4.1. In order to consider the energy consumption in the network, we define another variant of the problem, called $P_{\text {energy }}$, in Section 4.1 .

4.1. Solution Method for MMF SINR Link Computing. The MMFSLT problem aims to find a transmission power assignment scheme such that the concurrent transmissions in the network have a fair quality in terms of SINR. In order to achieve this goal, the first step is to maximize the minimum SINR value. However, it does not guarantee a MMF SINR for all the competitive links. More explicitly, the SINR value measured at all the receivers is not necessarily the same. Here, we go beyond this level and find the optimal max-min fair SINR for the set of competitive links. The basic steps for solving this problem are described in Algorithm 1.

Each step of Algorithm 1 is intended to find the $z$ value that represents the max-min SINR value among all transmission links in a given set $L$. The respective link is identified, and the $z$ value is allocated to its SINR value. Next, the algorithm removes the link from the set of links $L$ and continues with the remaining ones. It stops iterating when $z$ value achieves the $\beta$ threshold or there is no link anymore in the set $L$. As a first step we define a problem called $D_{1}$. The $z$ value is computed based on Algorithm 2. For the rest of the SINR values, we formulate and solve the problem $D_{k}$.

4.1.1. Formulating and Solving Problem $D_{1}$. The problem of maximizing the minimum value of SINR for a set of competitive links is modeled below:

$$
\begin{array}{ll}
\text { maximize } & \min _{j \in \mathrm{RX}} \mathrm{SINR}_{j}, \\
\text { s.t.: } & \mathrm{SINR}_{j} \geq \alpha \quad \forall j \in \mathrm{RX}, \\
& \frac{P_{i}}{\omega_{i j}} \geq \mathrm{RSSI}_{0} \quad \forall i \in \mathrm{TX}, \quad(i, j) \in L, \\
& P_{\min } \leq P_{i} \leq P_{\max } \quad \forall i \in \mathrm{TX},
\end{array}
$$

where the first constraint (6) guarantees that the minimum SINR value is beyond the lower $(\alpha)$ threshold. The second one (7) ensures that the signal in the receiver is sufficiently high for being detected and processed. And the third emphasizes the fact that the node's power values should be in the interval $\left[P_{\min }, P_{\max }\right]$.

In the above formulation, we redefine the objective function. We denote by $z$ the minimum value of $\operatorname{SINR}_{j}$ and 
add the respective constraints. Finally, the objective and the constraints of the problem $D_{1}$ are given as follows:

$$
\begin{array}{ll}
\operatorname{maximize} & z, \\
\text { s.t.: } \quad & \frac{P_{i} / \omega_{i j}}{\sum_{k \in \mathrm{TX} \backslash\{i\}}\left(P_{k} / \omega_{k j}\right)+N_{a}} \geq z \quad \forall(i, j) \in L, \\
& \alpha \leq z, \\
& \frac{P_{i}}{\omega_{i j}} \geq \mathrm{RSSI}_{0} \quad \forall j \in \mathrm{RX},(i, j) \in L \\
& P_{\min } \leq P_{i} \leq P_{\max } \quad \forall i \in \mathrm{TX} .
\end{array}
$$

For this problem, the variables are given by $P_{i}$ and $z$. We notice that the first constraint (10) is a nonlinear inequality. Nevertheless, we can easily handle this by fixing $z$ to some lower bounds and keep increasing it appropriately until it reaches the optimal solution. We begin by initially setting $z=\alpha$ in the first constraint. Because $\alpha$ is a lower bound for $z$, this assumption leads to a feasible solution (if such a solution exists for the initial problem). By assuming $z$ is a constant we obtain a LP model, the $D_{1}^{\prime}$ which is formulated as follows:

$$
\begin{array}{ll}
\operatorname{maximize} & \epsilon, \\
\text { s.t.: } \quad & \frac{P_{i}}{\omega_{i j}}-z \cdot\left(\sum_{k \in \mathrm{TX} \backslash\{i\}}\left(\frac{P_{k}}{\omega_{k j}}\right)+N_{a}\right) \geq \epsilon \\
& \alpha \leq z, \\
& \frac{P_{i}}{\omega_{i j}} \geq \mathrm{RSSI}_{0} \quad \forall j \in \mathrm{RX},(i, j) \in L, \\
& P_{\min } \leq P_{i} \leq P_{\max } \quad \forall i \in \mathrm{TX} .
\end{array}
$$

In constraint (15) (of the problem $D_{1}^{\prime}$ ), we have introduced the variable $\epsilon$ which will be helpful in increasing the $z$ value (for the sake of simplicity we use the same notation $z$ for both initial variable value and the current lower bound of $z$ which is updated (increased) constantly through iterations). Thus, $\epsilon$ is such that for each sender $i$ and receiver $j$

$$
\frac{\epsilon}{\sum_{k \in \mathrm{TX} \backslash\{i\}}\left(P_{k} / \omega_{k j}\right)+N_{a}}
$$

measures the gap between the $\operatorname{SINR}_{j}$ and the current $z$ value. Hence, by increasing $z$ according to (20) we ensure that there will be a feasible solution for the updated value of $z$ :

$$
\min _{j \in \mathrm{RX}} \frac{\epsilon}{\sum_{k \in \mathrm{TX} \backslash\{i\}}\left(P_{k} / \omega_{k j}\right)+N_{a}} .
$$

Indeed, this is true since the last solution remains feasible for the updated $z$. The idea behind this is to gradually increase the $z$ value until we reach its maximum value. Algorithm 2 describes these operations.

More precisely, the algorithm begins by solving the $D_{1}^{\prime}$ problem as defined above (with $z$ set to $\alpha$ ) and as a result we obtain the $P_{i}$ values (or the power values assigned to sender nodes). The ratios between $\epsilon$ and interference at each receiver (see formula (20)) are used to obtain the minimum value that allows increasing the SINR values while guaranteeing a feasible solution. The process is finite and the algorithm will stop either when $\epsilon$ becomes practically 0 or when the inferior bound of SINR becomes larger than $\beta$. In the latter case we set $z=\beta$; otherwise we take the last $z$ value. At this stage we cannot say too much on the theoretical complexity of the above algorithm. However, the methods perform quite well in practice and the process converges after a few steps. Notice last that, as suggested by an anonymous reviewer, the binary search could be a potential alternative method for computing the $z$ value. Preliminary tests have not been concluding so we stuck to the epsilon method.

4.1.2. Formulating and Solving Problem $D_{k}$. Similarly to problem $D_{1}$, the problem $D_{k}$ can be formulated as follows:

$$
\begin{array}{ll}
\operatorname{maximize} & z, \\
\text { s.t.: } & \frac{P_{i} / \omega_{i j}}{\sum_{k \in \mathrm{TX} \backslash\{i\}}\left(P_{k} / \omega_{k j}\right)+N_{a}} \geq z \\
\forall(i, j) \in K, & P_{i} / \omega_{i j} \\
& \frac{P_{k \in \mathrm{TX} \backslash\{i\}}\left(P_{k} / \omega_{k j}\right)+N_{a}}{\sum_{i}} \geq s_{(i, j)} \\
& \forall(i, j) \in M \backslash K, \\
& \omega_{i j} \mathrm{RSSI}_{0} \quad \forall j \in \mathrm{RX},(i, j) \in M, \\
& P_{\min } \leq P_{i} \leq P_{\max } \quad \forall i \in \mathrm{TX} .
\end{array}
$$

In comparison with $D_{1}$, the above formulation differs in two points. First, constraints (10) give rise to two types of constraints, that is, (23) and (22), with respect to links with SINR already computed and the others. Second, constraint (11) is not useful any more as we have increasing values of $z$. Hence, any $z$ solution to $D_{k}$ is necessarily larger equal to precedent $z$ and consequently to $\alpha$. Problem $D_{k}$ is solved in a similar way to problem $D_{1}$. More precisely, we use Algorithm 2 to solve $D_{k}$. To this end, we write an epsilon formulation, $D_{k}^{\prime}$, which is very similar to $D_{1}^{\prime}$.

4.1.3. Identifying SINR Constrained Links. With respect to Algorithm 1, once Algorithm 2 has reached $\epsilon=0$ and computed $z$, we need to find some links with SINR that cannot take higher value than $z$. Let us look in detail at problem $D_{1}$ and similar reasoning will hold for any $D_{k}$. Given problem (15)-(18) an easy way to find if a link is SINR constrained is to check if its dual coefficient of (15), let us 
say $\gamma_{(i, j)}$, is strictly positive. Indeed, using the complementary slackness property of duality theory, we have

$$
\begin{aligned}
\left(\frac{P_{i}}{\omega_{i j}}\right. & \left.z \cdot\left(\sum_{k \in \mathrm{TX} \backslash\{i\}}\left(\frac{P_{k}}{\omega_{k j}}\right)+N_{a}\right)-\epsilon\right) \gamma_{(i, j)} \\
& =\left(\frac{P_{i}}{\omega_{i j}}-z \cdot\left(\sum_{k \in \mathrm{TX} \backslash\{i\}}\left(\frac{P_{k}}{\omega_{k j}}\right)+N_{a}\right)\right) \gamma_{(i, j)}=0
\end{aligned}
$$

and we can say that link $(i, j)$ is SINR constrained if $\gamma_{(i, j)}>0$. Furthermore, there is at least one strictly positive value, since from the dual formulation of the above problem we have $\sum_{(i, j) \in L} \gamma_{(i, j)} \geq 1$.

4.2. Correctness of MMF SINR Computation. Without loss of generality we assume that all $z$ values computed during the algorithm are all less than $\beta$. The correctness of the proposed approach, that is, the optimality of the solution obtained by our iterative algorithm, is not obvious. Indeed, different transmission power assignments may satisfy all the constraints specified for the problem $D_{i}$ at each step $i$ of the algorithm. Furthermore, several links might possibly achieve the same SINR signal. Recall also that each step of Algorithm 2 yields at least one transmission link and the corresponding SINR value $(z)$ with respect to the maxmin fair SINR link transmissions. Once computed, this value is fixed and used as a constant for the remaining calculations. Questions naturally arise. How does this impact the upcoming $z$ value and consequently the quality of the computed assignment? Which link should be chosen and what are the consequences for the desired power transmission assignment? In the following we will try to answer these questions and prove formally the optimality of the max-min fair assignment computed by Algorithm 1 .

Theorem 1. The power assignment solution obtained at the end of the kth step of Algorithm 1 is such that there is no other power assignment that would allow the SINR value of transmission links in $L_{k}$ to be increased at the expense of other links with better SINR.

Proof. We prove this by mathematical induction on the number of steps of Algorithm 1. Obviously the statement holds for the first step of the algorithm. Indeed, the way the set $L_{1}$ is defined makes the existence of some other solutions achieving better SINR for $L_{1}$ impossible. We will prove now that if the above property is true for any step $k-1$ then it is also true for the following step. Hence, by recurrence hypothesis we assume that there is no way of increasing any SINR in $L_{(k-1)}$ by decreasing SINR of the other links. At this stage we notice that any solution obtained at the end of step $k$ is also a solution for all problems $D_{i}, i<k$, and all constraints (23) with respect to links in $M \backslash K$ are also satisfied at equality. Let us now consider some constrained link at step $k$; let us say $(p, q)$. With respect to the formulation of the corresponding problem $D_{k}^{\prime}$ and bearing in mind that $\epsilon=0$ and constraint (22) for link $(p, q)$ is tight, it is clear that there can be no way of increasing the SINR value for link $(p, q)$ simply by decreasing the SINR of some nonconstrained links (which are the only remaining links offering better SINR). Furthermore, this holds for all links in $M \backslash K$ as the current solution is as well a solution for all problems $D_{i}, i<k$, and the recurrence hypothesis applies. There is therefore no room to increase the SINR at this link and potential other links in $L_{k}$ with the same SINR, which concludes the proof of the theorem.

An immediate corollary of the above theorem is that the SINR values obtained at the end of Algorithm 1 give necessarily a max-min fair vector, since the result also holds for the solution obtained at the final step of the algorithm when all links are constrained.

4.3. Considering the Energy Consumption in the Network. The problem of MMFSLT computation aims to guarantee a "good" transmission medium for all concurrent links in the network. Hence, its focus falls upon the quality of links. In the WSN's context, energy is also considered as a relevant issue. Therefore, we can further process the results of Algorithm 1 in terms of economy of energy. By defining as objective the minimization of the sum of the nodes' power value, we formulate the problem $P_{\text {energy }}$ as follows:

$$
\begin{array}{ll}
\text { minimize } & \sum_{i \in \mathrm{TX}} P_{i}, \\
\text { s.t.: } \quad & \frac{P_{i}}{\omega_{i j}}-s_{(i, j)} \cdot\left(\sum_{k \in \mathrm{TX} \backslash\{i\}}\left(\frac{P_{k}}{\omega_{k j}}\right)+N_{a}\right) \geq 0 \\
& \forall(i, j) \in M, \\
& \frac{P_{i}}{\omega_{i j}} \geq \mathrm{RSSI}_{0} \quad \forall j \in \mathrm{RX}, \quad(i, j) \in M, \\
& P_{\text {min }} \leq P_{i} \leq P_{\max } \quad \forall i \in \mathrm{TX},
\end{array}
$$

where the first constraint guarantees that the SINR value in each receiver is bigger than the $s_{(i, j)}$ threshold $\left(s_{(i, j)}\right.$ in this case is considered a constant and it belongs to the $S$ vector of the MMF values). The other constraints are identical with problem $D_{k}$. Nevertheless, notice that in practice there is no much space left for modifying power assignment when SINR values are determined for all active links. One way to deal with it stands in satisfying the SINR constraint only for the first level of MMF.

\section{MMFSLT Problem for Time-Constant Transmission Power}

In this section, we examine the case of MMFSLT problem with time-constant transmission powers. For a given network $G=(V, E)$, the transmission links $E$ are allocated to different time slots with the same conditions as discussed in Section 3.2. The MMF algorithm (Algorithm 1) allocates the power to the transmitting nodes to guarantee MMF SINR at receiver for each given time slot. Hence, a node may have different transmission power depending on the time slot in which it transmits. Therefore, we formulate the following 
Input: A set $L$ of links located arbitrarily in the Euclidean plane;

Output: A feasible schedule $S$, the SINR $_{\text {threshold; }}$;

(1) while (there are still links not assigned to a slot) do

(2) $\Rightarrow$ take a new slot time;

(3) $\Rightarrow$ examine the non assigned links according to increasing distance to BS;

(4) $\Rightarrow$ assign link $(j)$ to the current time slot if the function test $(j)$ returns True;

(1) test $(j)$;

(2) cond1: the link $(j)$ and the other links in the current time slot have no receiver in common;

(3) cond2: the sender node of link $(j)$ and the receiver nodes of the other links in the current time slot are different;

(4) cond3: assign a power for each sender node by solving problem $D_{1}$. The minimal value of the SINR evaluated for the set of links belonging to the current time slot, including link $(j)$, is bigger than the $\operatorname{SINR}_{\text {threshold; }}$;

(5) if (cond1 \& cond 2 \& cond 3 ) then

(6) return True;

(7) else

(8) return False;

Algorithm 3: The principle of the algorithm for the JLSPA problem.

problem which seeks to find a unique transmission power for each node $\in V$ that is independent of the time slot and that ensures the SINR fairness. Let us denote by $T$ the whole frame divided in time slots, $\mathrm{RX}_{t}$ the set of receiving nodes at slot $t$, $\mathrm{TX}_{t}$ the set of transmitting nodes at slot $t$, and $M_{t}$ the set of activated links at slot $t$. An approach similar to the MMF algorithm can be given for the time-constant transmission power case at this stage: we report below the formulation of the counterpart of problem $D_{1}$ :

$$
\begin{array}{ll}
\operatorname{maximize} & \min \mathrm{SINR}_{t j} \quad j \in \mathrm{RX}_{t}, t \in T, \\
\text { s.t.: } \quad & \frac{P_{i}}{\omega_{i j}}-\alpha \cdot\left(\sum_{k \in \mathrm{TX}_{t} /\{i\}}\left(\frac{P_{k}}{\omega_{k j}}\right)+N_{a}\right) \geq 0, \\
& (i, j) \in M_{t}, \quad t \in T, \\
& \frac{P_{i}}{\omega_{i j}} \geq \mathrm{RSSI}_{0} \quad j \in \mathrm{RX}_{t}, \quad(i, j) \in M_{t}, t \in T, \\
& P_{\min } \leq P_{i} \leq P_{\max } \quad \forall i \in \mathrm{TX} .
\end{array}
$$

\section{The JLSPA Problem}

Finally, we deal with the JLSPA problem. For solving the problem we need to perform the following tasks.

(1) Identify the activated links.

(2) Design a scheduling scheme.

(3) Assign the transmissions power.

6.1. Network Topology and Scheduling Algorithm. Here, we assume an uplink traffic in the network, according to a well-defined routing scheme (see Figure 2). Each sensor aggregates the data during the relaying (converge-cast with

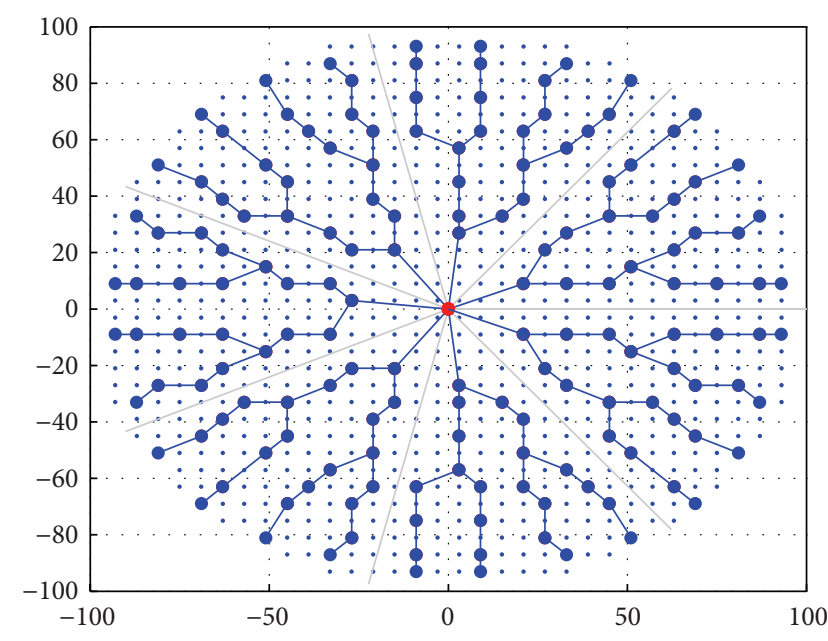

FIGURE 2: Routing tree: blue dots are the sensor nodes, and the red dot is the gateway.

data aggregation); therefore each transmission link may be activated only once. TDMA type of protocols following the same assumption is detailed in [28-30]. However, activating all the transmission links simultaneously may normally lead to unfeasible scenarios under SINR constraint.

Regarding the scheduling, it can be modeled as the oneshot scheduling problem in [16], assuming that the links weight will be equal to one unit. As this problem is shown to be $\mathscr{N} \mathscr{P}$-hard, we propose a bottom-up approach described in Algorithm 3. This approach is a greedy heuristic which intends to put in a time slot the maximum number of links such that the SINR constraint is respected (where SINR $_{\text {threshold }}$ represents the $\alpha$ value; see (2)). The set of links $L$ that need to be scheduled is given by solving the network configuration problem, as stated previously. In the set $L$ 
the links are sorted according to their respective coronas, meaning first we have the links of the corona closer to BS and next it will use the links of the second corona and so on. For each time slot, we try to put the links by beginning from those closer to the BS. The number of links that can be placed in a given time slot will be controlled by the three conditions given in lines 2-4 of the test() function. The algorithm proceeds by taking into consideration each link that has not been assigned to a time slot. If the function test() returns True, the link in consideration can be assigned to the current slot; otherwise the algorithm will check its validity for the next time slot.

The heart of this algorithm is the SINR computation (see condition 3) which corresponds to the third task, the power assignment strategy. For this we solve problem $D_{1}$; that is, we check the feasibility for a set of active links and compute the max-min SINR among them. Note that in the above approach we do not need to compute MMF SINR; nevertheless this can be done once the active links in a slot are determined. At this point one important question holds: how will the transmission power assignment strategy affect the schedule length? This question is answered in the following section.

6.2. Numerical Results for the JLSPA Problem. We apply two different power assignment strategies, (i) the linear power assignment and (ii) the power strategy for fair link transmissions (called MMF SINR strategy below), to the scheduling algorithm proposed previously. The linear power assignment strategy consists in assigning a power to each activated link, which is proportional to the link weight.

To compute the link weights we use the log-distance path loss model. This model is formally expressed according to

$$
\omega_{i j}\left(d_{i j}\right)=\mathrm{PL}_{0}+10 \cdot \gamma \cdot \log _{10} \frac{d_{i j}}{d_{0}}+N(0, \sigma),
$$

where $d_{i j}$ is the distance between transmitter $(i)$ and receiver $(j), d_{0}$ a reference distance, $\mathrm{PL}_{0}$ the power decay corresponding to $d_{0}, \gamma$ the path loss exponent (rate at which signal decays), and $N(0, \sigma)$ a normal (or Gaussian) random variable with mean 0 and variance $\sigma$, reflecting the attenuation (in $\mathrm{dB}$ ) caused by flat fading. The value of weight $\omega_{i j}$ is given in $\mathrm{dBm}$. The link weights are computed according to the model given in (35), but for computation simplicity we have not considered the $N(0, \sigma)$ value. For the rest of parameters, the reader can refer to Table 1 .

The MP parameters in Table 1 refer to the coefficients of MP presented in Section 4. For the $\alpha$ value we refer to the cochannel rejection ratio (CCRR) defined in the transceiver data sheets. From the empirical experiments provided in [31] we observed that the $\beta$ value can be approximated at $10 \mathrm{~dB}$. $\mathrm{RSSI}_{0}, P_{\min }$, and $P_{\max }$ are extracted from the sensor (MICAz) data sheet.

Our algorithm is coded in C++ using the CPLEX 12.1 Library. The program is compiled with MSVC in a Windows environment, and all experiments were conducted on an AMD Opteron $2.60 \mathrm{GHz}$.

We have applied the linear and the fair power strategy to the scheduling problem and computed the schedule length for cases when the SINR threshold varies. These results are
TABLE 1: Simulation parameters.

\begin{tabular}{lcc}
\hline Type & Parameter & Value \\
\hline & $\alpha$ & $1.99(3 \mathrm{~dB})$ \\
& RSSI $_{0}$ & $-90 \mathrm{dBm}$ \\
MP parameters & $P_{\min }$ & $-25 \mathrm{dBm}$ \\
& $P_{\max }$ & $0 \mathrm{dBm}$ \\
& $\beta$ & $10 \mathrm{~dB}$ \\
& $E_{\mathrm{rr}}$ & $10^{-7}$ \\
\hline \multirow{3}{*}{ Channel parameters } & $\gamma$ & 2 \\
& Reference distance $d_{0}$ & $1 \mathrm{~m}$ \\
& Power PL & 52.4 \\
\hline \multirow{3}{*}{ Radio parameters } & Noise floor & $-110 \mathrm{dBm}$ \\
& White Gaussian noise $N_{w}$ & $4 \mathrm{~dB}$ \\
& N $(0, \sigma)$ & 0 \\
\hline \multirow{2}{*}{ Network topology instance } & Internodes distance & $6 \mathrm{~m}$ \\
& Number of activated links & 161 \\
\hline
\end{tabular}

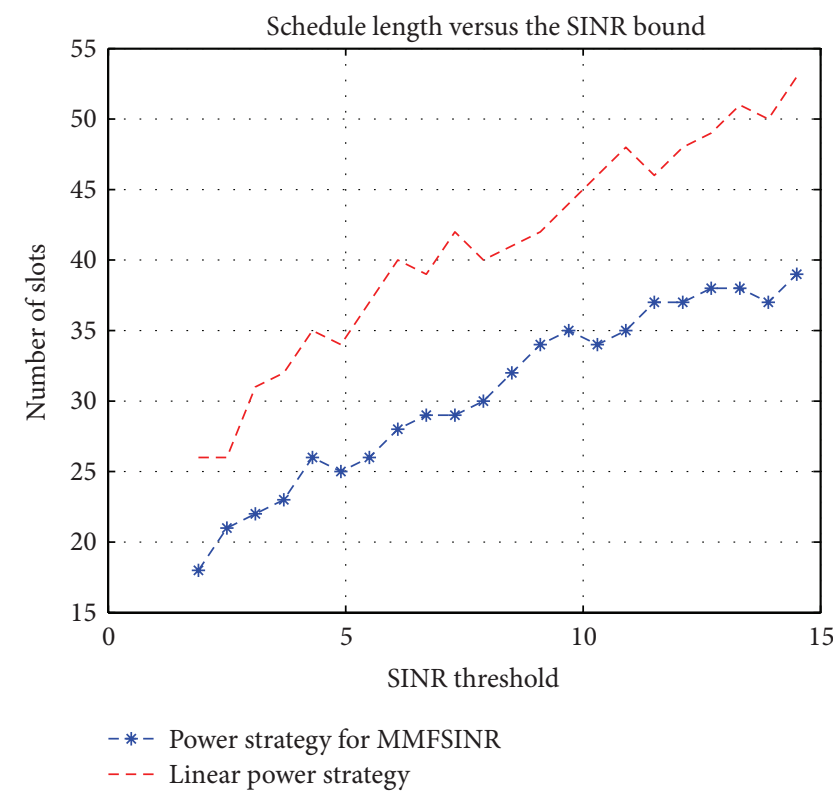

FIGURE 3: Time slots number versus SINR.

presented in Figure 3. The fair power assignment strategy improves the schedule length by at least $31 \%$ (fair power assignment strategy requires 18 time slots with respect to linear schedule length which needs 26 time slots for a SINR threshold equal to 1.9 units). As we can observe, the number of slots required to schedule all the links has the tendency to increase for bigger values of SINR thresholds.

Based on the above limited numerical results, it seems that the fair power assignment strategy can be helpful in reducing the schedule length. However, an extended numerical study would be necessary to confirm the findings while there is still place for further improving the scheduling heuristic. 


\section{Future Work}

We formulated and solved the max-min fair SINR link transmission problem which consists in allocating power transmission to nodes such that the SINR values of active links are MMF. This problem is solved optimally and, to the best of our knowledge, we are the first to propose an exact method. By implementing our method to JLSPA problem, we show that the schedule length may be significantly reduced. Moreover, the design of a cooperative approach provides a fair medium for the concurrent links and, therefore, the best possible scenario for having successful transmissions. We extended the problem also to the case when the transmission power of nodes is constant through the time frame. However, different problems may be interesting to be investigated as future work, such as

(i) improving the scheduling algorithm in order to have lower bounds for the JLSPA problem,

(ii) developing an adaptive transmission power control algorithm that operates in a distributed manner; the algorithm should be able to adapt its transmission power quickly to suit a rapidly changing radio environment.

\section{Conflict of Interests}

The authors declare that there is no conflict of interests regarding the publication of this paper.

\section{Acknowledgment}

Part of this work has been performed in the AgentschapNL project Reconsurve-NL.

\section{References}

[1] D. Son, B. Krishnamachari, and J. Heidemann, "Experimental study of the effects of transmission power control and blacklisting in wireless sensor networks," in Proceedings of the 1st Annual IEEE Communications Society Conference on Sensor and Ad Hoc Communications and Networks (SECON '04), pp. 289298, October 2004.

[2] S. Lin, J. Zhang, G. Zhou, L. Gu, J. A. Stankovic, and T. He, "ATPC: adaptive transmission power control for wireless sensor networks," in Proceedings of the 4th International Conference on Embedded Networked Sensor Systems (SenSys '06), pp. 223-236, November 2006.

[3] G. Hackmann, O. Chipara, and C. Lu, "Robust topology control for indoor wireless sensor networks," in Proceedings of the 6th ACM Conference on Embedded Networked Sensor Systems (SenSys '08), pp. 57-70, November 2008.

[4] A. Gogu, D. Nace, A. Dilo, and N. Meratnia, "Review of optimization problems in wireless sensor networks," in Telecommunication Networks, Current Status and Future Trends, J. H. Ortiz, Ed., chapter 7, Intech, 2012.

[5] S. Chen, Y. Fang, and Y. Xia, "Lexicographic maxmin fairness for data collection in wireless sensor networks," IEEE Transactions on Mobile Computing, vol. 6, no. 7, pp. 762-776, 2007.
[6] X. Wang, K. Kar, and J.-S. Pang, "Lexicographic max-min fair rate allocation in random access wireless networks," in Proceedings of the 45th IEEE Conference on Decision and Control (CDC '06), pp. 1284-1290, December 2006.

[7] A. Sridharan and B. Krishnamachari, "Maximizing network utilization with max-min fairness in wireless sensor networks," in Proceedings of the 5th International Symposium on Modeling and Optimization in Mobile, Ad Hoc, and Wireless Networks (WiOpt '07), pp. 1-9, April 2007.

[8] L. Tassiulas and S. Sarkar, "Maxmin fair scheduling in wireless networks," in Proceedings of the Annual Joint Conference on the IEEE Computer and Communications Societies (INFOCOM '02), pp. 763-772, June 2002.

[9] D. Nace and M. Pióro, "Max-min fairness and its applications to routing and load-balancing in communication networks: a tutorial," IEEE Communications Surveys and Tutorials, vol. 10, no. 4, pp. 5-17, 2008.

[10] T. A. Al-Khdour and U. Baroudi, "An energy-efficient distributed schedulebased communication protocol for periodic wireless sensor networks," Arabian Journal for Science and Engineering, vol. 35, no. 2, pp. 153-168, 2010.

[11] S. C. Ergen and P. Varaiya, "TDMA scheduling algorithms for wireless sensor networks," Wireless Networks, vol. 16, no. 4, pp. 985-997, 2010.

[12] S. Gandham, M. Dawande, and R. Prakash, "Link scheduling in sensor networks: distributed edge coloring revisited," in Proceedings of the 24th Annual Joint Conference on the IEEE Computer and Communications Societies (INFOCOM '05), vol. 4, pp. 2492-2501, March 2005.

[13] R. Kawano and T. Miyazaki, "Distributed data aggregation in multi-sink sensor networks using a graph coloring algorithm," in Proceedings of the 22nd International Conference on Advanced Information Networking and Applications Workshops (AINA '08), pp. 934-940, March 2008.

[14] D. Chafekar, V. S. A. Kumar, M. V. Marathe, S. Parthasarathy, and A. Srinivasan, "Approximation algorithms for computing capacity of wireless networks with SINR constraints," in Proceedings of the 27th Annual Joint Conference on the IEEE Computer and Communications Societies (INFOCOM '08), pp. 1840-1848, April 2008.

[15] T. Moscibroda and R. Wattenhofer, "The complexity of connectivity in wireless networks," in Proceedings of the 25th Annual Joint Conference on the IEEE Computer and Communications Societies (INFOCOM '06), pp. 1-13, April 2006.

[16] O. Goussevskaia, Y. A. Oswald, and R. Wattenhofer, "Complexity in geometric SINR," in Proceedings of the 8th ACM International Symposium on Mobile Ad Hoc Networking and Computing (MobiHoc '07), pp. 100-109, September 2007.

[17] P. Santi, R. Maheshwari, G. Resta, S. Das, and D. M. Blough, "Wireless link scheduling under a graded SINR interference model," in Proceedings of the 2nd ACM International Workshop on Foundations of Wireless Ad Hoc and Sensor Networking and Computing (FOWANC '09), pp. 3-12, May 2009.

[18] T. Kesselheim, "A constant-factor approximation for wireless capacity maximization with power control in the SINR model," in Proceedings of the 21st Annual ACM-SIAM Symposium on Discrete Algorithms (SODA '11), pp. 1549-1559, 2011.

[19] Q. S. Hua, Scheduling wireless links with SINR constraints [Ph.D. thesis], The University of Hong Kong, 2009. 
[20] P. Gupta and P. R. Kumar, "Critical power for asymptotic connectivity in wireless networks," in Stochastic Analysis, Control, Optimization and Applications, pp. 547-566, Birkhäauser, Boston, Mass, USA, 1999.

[21] A. Fanghänel, T. Kesselheim, H. Räcke, and B. Vöcking, "Oblivious interference scheduling," in Proceedings of the ACM Symposium on Principles of Distributed Computing (PODC '09), pp. 220-229, 2009.

[22] A. Gogu, S. Chatterjea, D. Nace, and A. Dilo, "The problem of joint scheduling and power assignment in wireless sensor networks," in Proceedings of the 27th IEEE International Conference on Advanced Information Networking and Applications (AINA '13), pp. 348-355, March 2013.

[23] P. Björklund, P. Värbrand, and D. Yuan, "Resource optimization of spatial TDMA in ad hoc radio networks: a column generation approach," in Proceedings of the 22nd Annual Joint Conference on the IEEE Computer and Communications Societies (INFOCOM '03), vol. 2, pp. 818-824, April 2003.

[24] S. A. Borbash and A. Ephremides, "Wireless link scheduling with power control and SINR constraints," IEEE Transactions on Information Theory, vol. 52, no. 11, pp. 5106-5111, 2006.

[25] B. Katz, M. Völker, and D. Wagner, "Energy efficient scheduling with power control for wireless networks," in Proceedings of the 8th International Symposium on Modeling and Optimization in Mobile, Ad Hoc, and Wireless Networks (WiOpt '10), pp. 160-169, June 2010.

[26] M. Haenggi, J. G. Andrews, F. Baccelli, O. Dousse, and M. Franceschetti, "Stochastic geometry and random graphs for the analysis and design of wireless networks," IEEE Journal on Selected Areas in Communications, vol. 27, no. 7, pp. 1029-1046, 2009.

[27] A. Sridharan and B. Krishnamachari, "Max-min fair collisionfree scheduling for wireless sensor networks," in Proceedings of the 23rd IEEE International Performance, Computing, and Communications Conference (IPCCC '04), pp. 585-590, April 2004.

[28] S. Chatterjea, L. F. W. van Hoesel, and P. J. M. Havinga, "AILMAC: an adaptive, information-centric and lightweight MAC protocol for wireless sensor networks," in Proceedings of the Intelligent Sensors, Sensor Networks and Information Processing Conference (ISSNIP '04), pp. 381-388, Melbourne, Australia, December 2004.

[29] L. van Hoesel and P. Havinga, "A lightweight medium access protocol (LMAC) for wireless sensor networks," in Proceedings of the 1st International Workshop on Networked Sensing Systems (INSS '04), Tokyo, Japan, 2004.

[30] K. Langendoen, "Medium access control in wireless sensor networks," in Medium Access Control in Wireless Networks Volume II: Practice and Standards, chapter 20, pp. 535-560, Nova Science, 2008.

[31] M. Z. Zamalloa and B. Krishnamachari, "An analysis of unreliability and asymmetry in low-power wireless links," ACM Transactions on Sensor Networks, vol. 3, no. 2, Article ID 1240227, 2007. 


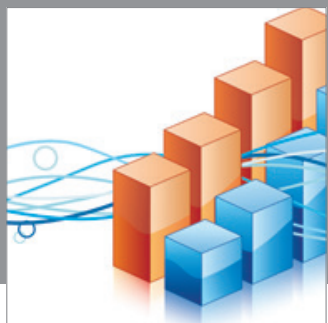

Advances in

Operations Research

mansans

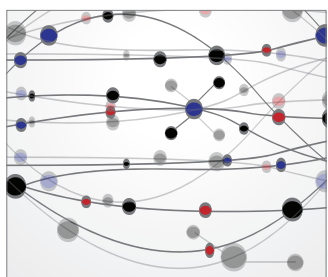

The Scientific World Journal
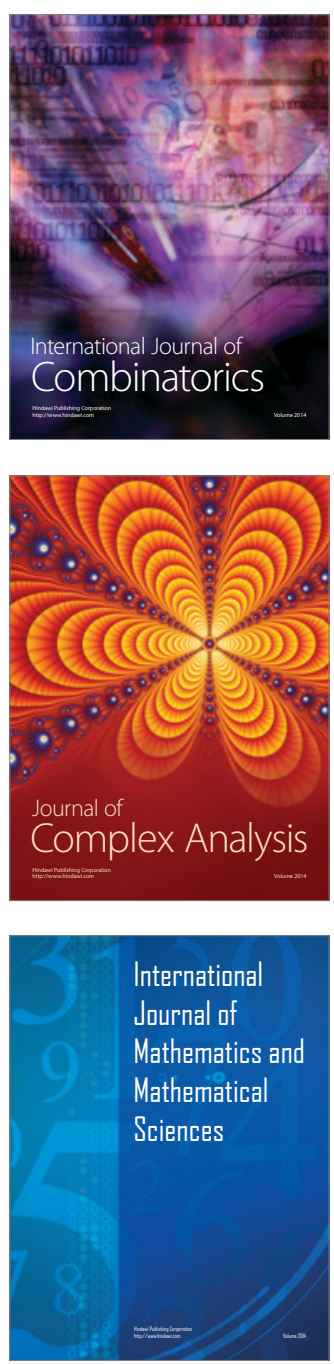
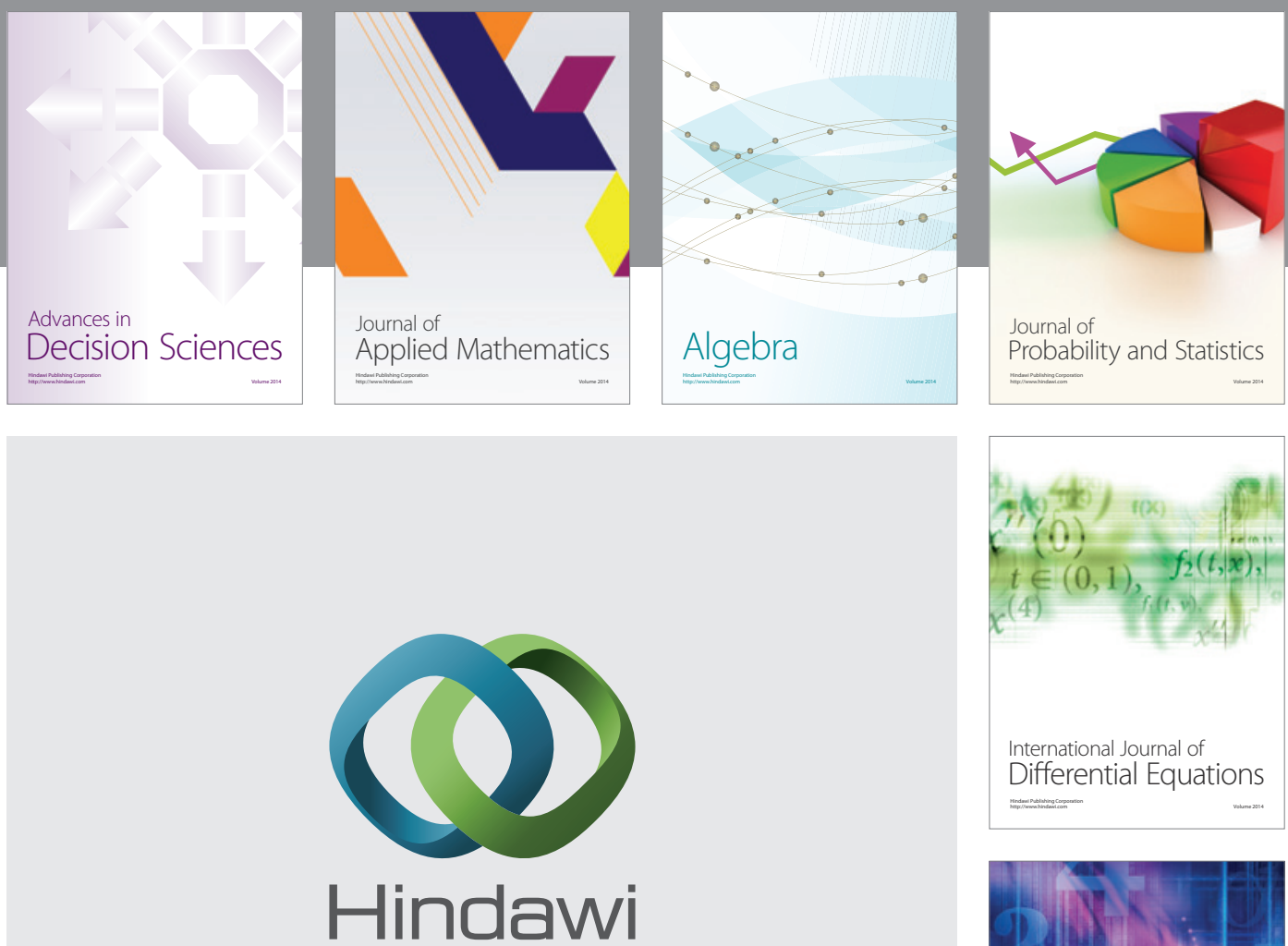

Submit your manuscripts at http://www.hindawi.com
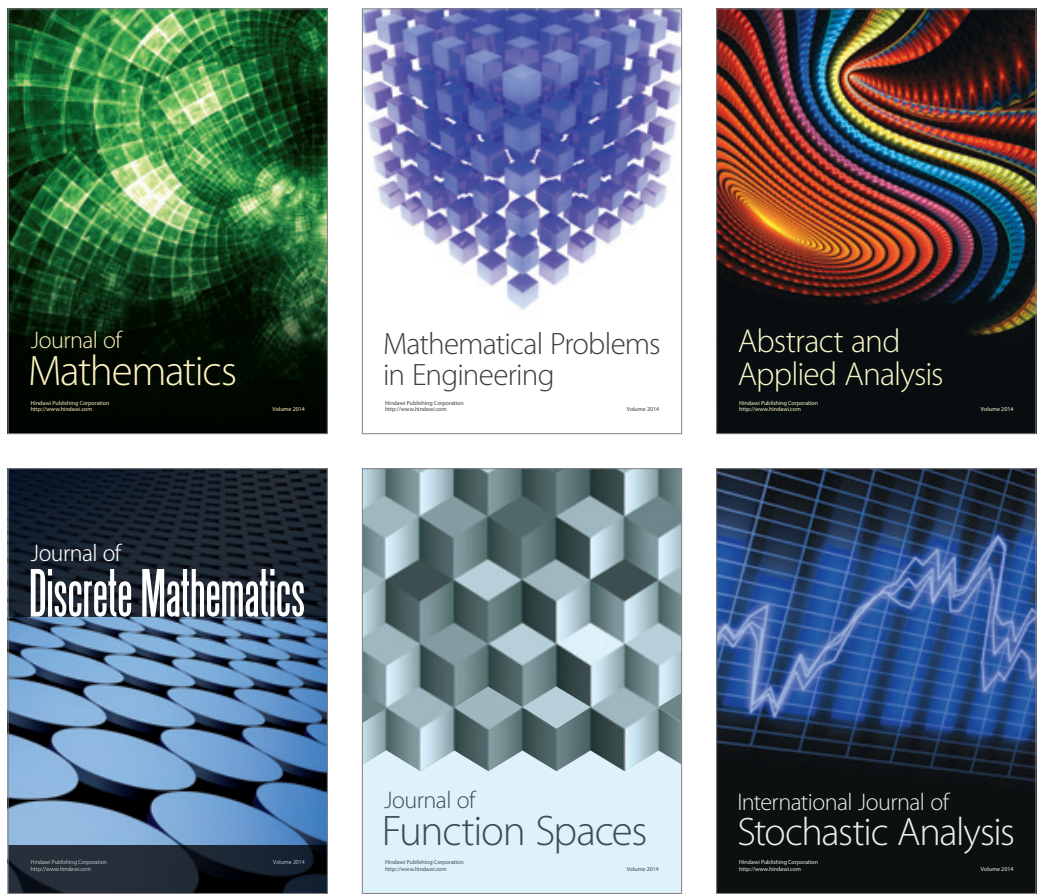

Journal of

Function Spaces

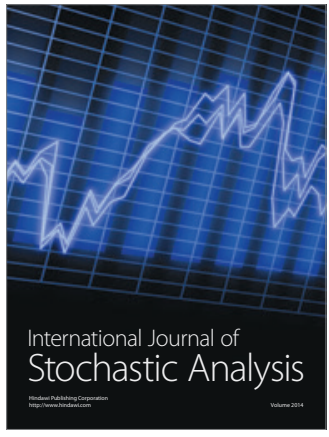

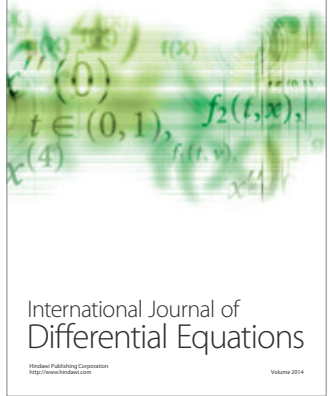
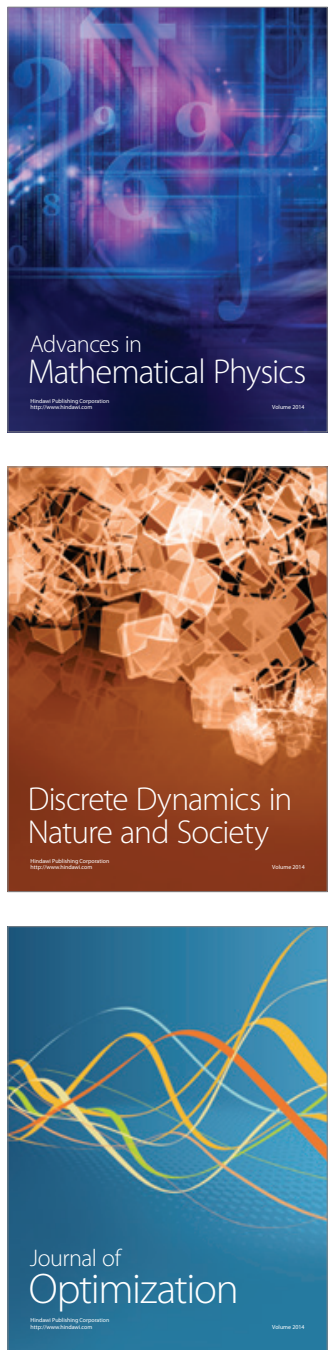